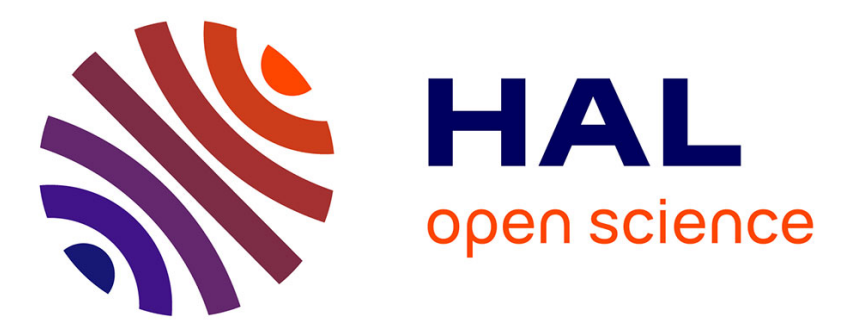

\title{
Condition Monitoring of Electrical Power Plant Components During Operational Transients
}

Piero Baraldi, Francesco Di Maio, Luca Pappaglione, Enrico Zio, Rédouane Seraoui

\section{- To cite this version:}

Piero Baraldi, Francesco Di Maio, Luca Pappaglione, Enrico Zio, Rédouane Seraoui. Condition Monitoring of Electrical Power Plant Components During Operational Transients. Proceedings of the Institution of Mechanical Engineers, Part O: Journal of Risk and Reliability, 2012, 226 (6), pp.568583. 10.1177/1748006X12463502 . hal-00765275

\section{HAL Id: hal-00765275}

https://hal-centralesupelec.archives-ouvertes.fr/hal-00765275

Submitted on 14 Dec 2012

HAL is a multi-disciplinary open access archive for the deposit and dissemination of scientific research documents, whether they are published or not. The documents may come from teaching and research institutions in France or abroad, or from public or private research centers.
L'archive ouverte pluridisciplinaire HAL, est destinée au dépôt et à la diffusion de documents scientifiques de niveau recherche, publiés ou non, émanant des établissements d'enseignement et de recherche français ou étrangers, des laboratoires publics ou privés. 


\title{
Condition Monitoring of Electrical Power
}

\section{Plant Components During Operational}

\section{Transients}

Piero Baraldi ${ }^{\mathrm{a},}{ }^{*}$, Francesco Di Maio ${ }^{\mathrm{a}}$, Luca Pappaglione ${ }^{\mathrm{a}}$, Enrico Zio ${ }^{\mathrm{b}, \mathrm{a}}$, Redouane Seraoui ${ }^{\mathrm{c}}$

\author{
${ }^{a}$ Dipartimento di Energia, Politecnico di Milano, via Ponzio 34/3, Milano, Italy \\ ${ }^{\mathrm{b}}$ Chair on System Science and the Energetic Challenge, European Foundation for New energy - Electricite de \\ France, Ecole Centrale, Paris and Supelec, Paris, France \\ ${ }^{c} E D F-R \& D \backslash S T E P$ Simulation et Traitement de l'information pour l'exploitation des systèmes de production, 6 \\ quai Watier, Chatou Cedex, France
}

\begin{abstract}
Monitoring the condition of a component is typically based on an empirical model that estimates the values of some measurable variables (signals) in normal conditions and triggers the fault alarm when the reconstruction deviates from the measured signal. When condition monitoring is performed during plant operational transients the intrinsically dynamic behavior of the signals should be taken into account. To this purpose, two approaches are proposed in this work. The former is based on the development of several reconstruction models, each one dedicated to a different operational zone of the component. The latter is based on the preprocessing of the signals by means of Haar wavelet transforms. The performance of the two proposed approaches are compared to that of the traditional reconstruction approach used for stationary conditions, with respect to a case study concerning the condition monitoring of a gas turbine during start-up transients.
\end{abstract}

\footnotetext{
*Corresponding author. E-mail: piero.baraldi@polimi.it, Tel. +39 022399 6355, Fax. +39 0223996309
} 
Keywords: Condition monitoring, Signal reconstruction, AAKR, Haar transform, Gas turbine, Start-up transients

\section{LIST OF SYMBOLS AND ACRONYMS}

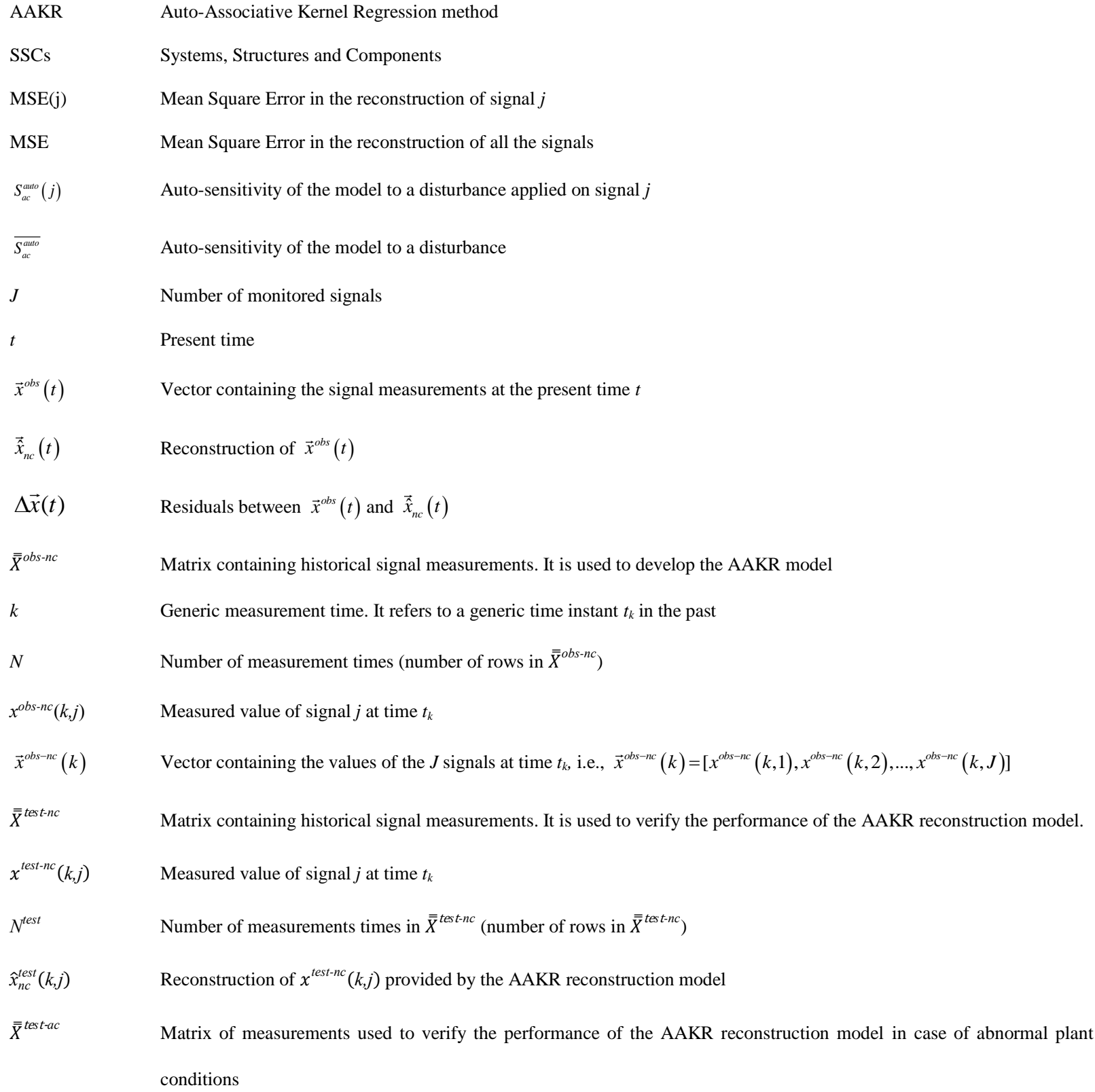

SSCs

Systems, Structures and Components

$\operatorname{MSE}(\mathrm{j})$

Mean Square Error in the reconstruction of signal $j$

MSE

Mean Square Error in the reconstruction of all the signals

$S_{a c}^{\text {auto }}(j)$

Auto-sensitivity of the model to a disturbance applied on signal $j$

Auto-sensitivity of the model to a disturbance

Number of monitored signals

Present time

$\vec{x}^{o b s}(t) \quad$ Vector containing the signal measurements at the present time $t$

$\vec{x}_{n c}(t) \quad$ Reconstruction of $\vec{x}^{o b s}(t)$

$\Delta \vec{x}(t) \quad$ Residuals between $\vec{x}^{o b s}(t)$ and $\overrightarrow{\hat{x}}_{n c}(t)$

$\overline{\bar{X}}^{o b s-n c} \quad$ Matrix containing historical signal measurements. It is used to develop the AAKR model

Generic measurement time. It refers to a generic time instant $t_{k}$ in the past

$N$

Number of measurement times (number of rows in $\overline{\bar{X}}^{\text {obs-nc}}$ )

$x^{o b s-n c}(k, j) \quad$ Measured value of signal $j$ at time $t_{k}$

$\vec{x}^{o b s-n c}(k) \quad$ Vector containing the values of the $J$ signals at time $t_{k}$, i.e., $\vec{x}^{o b s-n c}(k)=\left[x^{o b s-n c}(k, 1), x^{o b s-n c}(k, 2), \ldots, x^{o b s-n c}(k, J)\right]$

$\overline{\bar{X}}^{\text {test-nc }} \quad$ Matrix containing historical signal measurements. It is used to verify the performance of the AAKR reconstruction model.

$x^{\text {test-nc }}(k, j) \quad$ Measured value of signal $j$ at time $t_{k}$

$N^{\text {test }} \quad$ Number of measurements times in $\overline{\bar{X}}^{\text {test-nc }}$ (number of rows in $\overline{\bar{X}}^{\text {test }-n c}$ )

$\hat{x}_{n c}^{\text {test }}(k, j) \quad$ Reconstruction of $x^{\text {test-nc }}(k, j)$ provided by the AAKR reconstruction model

$\overline{\bar{X}}^{\text {testac }} \quad$ Matrix of measurements used to verify the performance of the AAKR reconstruction model in case of abnormal plant conditions

$x^{\text {test-ac }}(k, j) \quad$ Measured value of the signal $j$ at time $t_{k}$ during abnormal plant conditions 


\begin{tabular}{|c|c|}
\hline$\hat{x}^{\text {test }}(k, j)$ & Reconstruction of $x^{\text {test-ac }}(k, j)$ \\
\hline$R$ & Number of available operational transients in normal conditions \\
\hline$N_{r}$ & Number of measurements performed during operational transient $r$ \\
\hline$\overline{\bar{X}}_{r}^{o b s-n c}$ & Matrix of signal measurements collected during the $r$-th operational transient \\
\hline$x_{r}^{o b s-n c}\left(k_{r}, j\right)$ & Measured value of signal $j$ at time $t_{k r}$ during operational transient $r$ \\
\hline$w_{1}$ & Wavelet coefficient indicating the mean value of the signal, taken at the highest, i.e. coarsest, scale of the Haar transform \\
\hline$w_{2}$ & Maximum wavelet coefficient over all the scales of the Haar transform \\
\hline$w_{3}$ & Minimum wavelet coefficient over all the scales of the Haar transform \\
\hline$\vec{y}(t)$ & 3. $J$-dimensional vector whose features are the 3 Haar coefficients of each signal of $\vec{x}^{o b s}(t)$ \\
\hline $\overrightarrow{\hat{y}}(t)$ & Reconstruction of $\vec{y}(t)$ \\
\hline$T_{s}$ & Window size used for the Haar wavelet dyadic decomposition \\
\hline$h$ & Gaussian kernel bandwidth \\
\hline$w(k)$ & Weights associated to the generic $k$-th measurement vector of $\overline{\bar{X}}^{o b s-n c}$ by the AAKR reconstruction model \\
\hline$d^{2}(k)$ & Distance between the current measurement $\vec{x}^{o b s}(t)$ and $\vec{x}^{o b s-n c}(k)$ \\
\hline
\end{tabular}

\section{Introduction}

To maintain for long periods of time a high level of performance and reliability of electrical power systems, structures and components (SSCs), faults and abnormalities in operation must be detected promptly [1-5]. This requires the development of efficient condition monitoring techniques to detect signal changes indicative of an incipient failure. Monitoring the condition of a component is typically based on an empirical model estimating the values of measurable variables (signals) in normal conditions. During operation, the measured values are compared with those estimated ('reconstructed') by the model: a deviation between the observed and reconstructed values reveals the presence of an abnormal condition [1].

This work focuses on the issue of performing condition monitoring during operational transients such as start-up and shut-down. The original contribution consists in the development of two different approaches for signal reconstruction during operational plant transients and in the comparison of their performances. Once the signal reconstruction task is performed, statistical methods of analysis of the deviations between the observed and recon- 
structed values, such as the well known Sequential Probability Ratio Test (SPRT), can be applied for fault detection and alarm triggering. The application of these latter methods is not object of the present work.

The first proposed approach for signal reconstruction is based on the development of different reconstruction models, each one dedicated to a different plant operational zone. In the second approach, the signal reconstruction takes into account not only the present value but also the past evolution of the signals. To this purpose, the Haar wavelet transform [6-8] is applied to the signal measurements taken in a proper time window, and three wavelet coefficients are selected for characterizing each signal behavior within the time window considered [9-11]; then, the reconstruction model is applied to the obtained wavelet coefficients.

The empirical reconstruction model used in the present analysis is the Auto-Associative Kernel Regression method (AAKR) [4,5], which uses for the reconstruction historical observations of the signals taken during normal transient operation.

The approaches are tested on a real case study concerning the monitoring of a gas turbine during start-up transients. The condition monitoring performance is evaluated with respect to metrics that measure $i$ ) accuracy, i.e., the ability of the overall model to correctly and accurately reconstruct the signal values when the plant is in normal operation; ii) robustness, i.e., the overall model ability to reconstruct the signal values in case of abnormal operation and consequent anomalous behavior of some monitored signals [5,12].

This work is organized as follows: Section 2 introduces the condition monitoring scheme, whereas Section 3 illustrates the signal reconstruction approaches developed for condition monitoring during operational transients. The application of the proposed approaches to the industrial case study concerning the monitoring of a gas turbine during start-up transients is discussed in Section 4. Finally, Section 5 draws the conclusions of the work.

\section{Condition Monitoring}

Figure 1 shows a typical scheme of condition monitoring of a component. During operation, we assume that at the current time $t$ a vector $\vec{x}^{\text {obs }}(t)$ containing the measurements of $J$ signals, $\vec{x}^{o b s}(t)=\left[x^{o b s}(1), x^{o b s}(2), \ldots, x^{o b s}(J)\right]$ becomes available. Notice that, for the sake of simplicity, the current time $t$ is not reported in the notation $x^{\text {obs }}(j)$ 
of the value of signal $j$ measured at time $t$. Vector $\vec{x}^{o b s}(t)$ is sent to an auto-associative empirical model of the component behavior during operational transients in normal conditions. Thus, the model provides in output the values of the input signals expected in case of normal conditions at the current time $t$, 䇾 $(t)=\left[x_{n c}(1), \mathfrak{X}_{n c}(), \ldots, \hat{X}_{n c}(J)\right]$. A deviation between the measured $\vec{x}^{o b s}(t)$ and reconstructed $\overrightarrow{\hat{x}}_{n c}(t)$ values in one or more signals reveals the presence of faults [1]. In other words, in case of operational transients in normal conditions, the measured values $\vec{x}^{o b s}(t)$ should be very similar to the model reconstructions $\overrightarrow{\hat{x}}_{n c}(t)$, whereas in case of operational transients in abnormal conditions the reconstructed $\overrightarrow{\hat{x}}_{n c}(t)$ differ from the measured $\vec{x}^{o b s}(t)$. Notice that one usually does not know whether the component is experiencing a transient in normal or abnormal conditions: by observing the vector of residuals $\Delta \vec{x}=\vec{x}^{o b s}(t)-\vec{X}_{n c}(t)=\left[x^{o b s}()-\mathfrak{X}_{n c}(), x^{o b s}(2)-\mathfrak{X}_{n c}(), \ldots, x^{o b s}(J)-\mathcal{X}_{n c}(J)\right]$, it is possible to detect the component condition during the transient. In this respect, several methods of analysis of the residuals $\Delta \vec{x}$ for fault detection and alarm triggering have been developed, e.g. the well known Sequential Probability Ratio Test (SPRT) [13-14].

\subsection{Auto-Associative Kernel Regression (AAKR) method}

The model considered in this work for reconstructing the component behavior during operational transients in normal conditions is based on the AAKR method [4] whose basic idea is to reconstruct the signal values at the current time $t$ in case of normal conditions, $\overrightarrow{\hat{x}}_{n c}(t)$, given a current signal measurement vector, $\vec{x}^{o b s}(t)$, as a weighted sum of historical observations. Thus, the application of the AAKR method requires the availability of a set of historical measurements performed at past times $t_{k}, k=1, \ldots, N$ which will be indicated by the matrix $\overline{\bar{X}}^{o b s-n c}$ whose generic element $x^{o b s-n c}(k, j)$ is the measured value of signal $j$ at time $t_{k}$.

Appendix A provides the details of the method.

Figure 1. Condition monitoring scheme. 


\subsection{Condition monitoring performance metrics}

In order to evaluate the performance of a condition monitoring model, the following metrics are considered:

1) The accuracy, i.e. the ability of the model to correctly and accurately reconstruct the signal values when the plant is in normal conditions. An accurate condition monitoring model allows reducing the number of false alarms, i.e. detections of faulty behaviors when no faulty conditions are actually occurring. The accuracy metric is typically defined as the Mean Square Error (MSE) between the model reconstruction and the signal measured values [4,5]. Appendix B provides the details for the accuracy metric computation.

2) The robustness, i.e. the model ability to reconstruct the values of the signals of interest during abnormal conditions when some monitored signals behave anomalously. In case of operational transients in abnormal conditions, a robust model reconstructs the value of a plant signal as if the plant were operating in normal conditions: then, the differences between the measured and the reconstructed signal values can easily identify the abnormal transient conditions. A quantitative indicator of robustness is the auto-sensitivity of the model to a disturbance $\left(\overline{S_{a c}^{\text {auto }}}\right)$. The computation of the metric, which is described in Appendix C, requires the availability of plant data collected during operational transients in abnormal conditions. Since, however, operational transients in abnormal conditions are usually rare, simulation is typically used to artificially inject abnormality by adding realistic deviations to the signals measured during operational transients in normal conditions. Typically, the signal value is altered with respect to its value in normal transients, by adding a Gaussian noise with mean zero and standard deviation proportional to:

- the standard deviation of the signal in the transients; this term which is computed by considering the historical measurements of the signal during operational transients, is introduced in order to take into account the fact that signals characterized by high variations during the transients are expected to be more sensible to abnormal conditions than signals characterized by small variations;

- the instantaneous value of the signal; this term takes into account the fact that the effect of an abnormal condition is usually directly related to the signal value [15]. 


\subsection{Cross-validation procedure for the estimation of the performance metrics}

In order to accurately estimate the values of the performance metrics and their confidence intervals on test sets formed by operational transients not previously used in the model development phase, a cross-validation procedure is adopted in the application that follows [16-18]. In particular, the so called "10-fold" cross-validation error estimate is used to compare the performances of different reconstruction approaches [19]. The transients in the original dataset are randomly partitioned into 10 blocks of equal size; one of these blocks is used as validation data subset for the evaluation of the performance metrics of interest, and the remaining 9 blocks are combined together to constitute the training data subset. The cross-validation process is then repeated 10 times (the 10 folds), each time using a different block as validation set.

\section{Signal reconstruction during transient conditions}

Sections 3.1, 3.2 and 3.3 describe the approaches considered in the present work for signal reconstruction during transient conditions. The reconstruction models are built assuming to have available the measurements of $J$ signals taken during $R$ transients in normal conditions. For the generic $r$-th transient, $r=1, \ldots, R$, signal measurements are taken at $N_{r}$ different times $t_{k_{r}}$ and they are encoded in the matrix $\overline{\bar{X}}_{r}^{\text {obs-nc }}$ whose generic element $x_{r}^{o b s-n c}\left(k_{r}, j\right), k=1, \ldots, N_{r}$ and $j=1, \ldots, J$, is the value of signal $j$ at time $t_{k_{r}}$ in the training transient $r$. All the available information is encoded in the matrix $\overline{\bar{X}}^{\text {obs-nc}}=\bigcup_{r=1}^{R}\left(\overline{\bar{X}}_{r}^{o b s-n c}\right)$ containing the total number $N=\sum_{r=1}^{R} N_{r}$ of available $J$-dimensional measurement vectors $\vec{x}^{o b s-n c}(k)$ which can be used to train the reconstruction models.

\subsection{Approach 0: a single reconstruction model}

A single AAKR reconstruction model can be built using all the available $N$ measurement vectors $\vec{x}^{o b s-n c}(k)$ in $\overline{\bar{X}}^{o b s-n c}$. In practice, the problem of signal reconstruction during transient conditions is addressed in the same way of the problem of signal reconstruction in stationary conditions, by developing a single AAKR reconstruction 
model built considering all the available information $\overline{\bar{X}}^{o b s-n c}$. This Approach 0 will be applied in the case study, to serve as reference for comparison.

\subsection{Approach 1: a dedicated reconstruction model for each operational zone of the component}

An operational zone is typically defined by the minimum and maximum values of a pivot signal which characterizes the component operation driving the evolutions of the other plant signals. If, for examples, we consider a plant start up, the turbine speed directly influence the evolutions of several other plant signals such as plant temperatures and pressures. The basic idea behind this approach is to develop a dedicated reconstruction model for each component operational zone. To this aim, the $N J$-dimensional measurement vectors $\bar{x}^{o b s-n c}(k)$ in $\overline{\bar{X}}^{o b s-n c}$ are split into different training sets, accordingly to the value of the pivot signal. Then, for each operational zone, an AAKR model is built using the corresponding training set.

Once the fault detection system has been built, it can be used for the fault detection during operational transients. To this aim, the value of the pivot signal in the current plant measurements $\vec{x}^{o b s}(t)$ is compared to the pivot minimum and maximum values in the different operational zones and is sent to the corresponding reconstruction model (Figure 2).

Figure 2. Scheme of the overall reconstruction model used in approach 1.

\subsection{Approach 2: reconstruction of the wavelet transform}

This approach is based on the use of a signal pre-processing tool [9-11] for the extraction of features catching the dynamics of the signal evolutions. The Haar wavelet transform [6] is applied on a sliding window of the actual signal time-series and, among all the obtained wavelet coefficients, the following are selected:

- $\quad$ the mean value of the signal, $w_{1}$, taken at the highest, i.e. coarsest, scale; 
- $\quad$ the maximum wavelet coefficient, $w_{2}$, over all the scales;

- the minimum wavelet coefficient, $w_{3}$, over all the scales.

The rationale behind this choice is that $w_{1}$ captures the general trend of the signal across the windows in a compact way, while $w_{2}$ and $w_{3}$ capture important variations in the signal within a single window (e.g. downward or upward trends, step changes, spikes, etc.) which would otherwise be severely smoothed out by the compression process [20]. The window size, $T_{s}$, is selected so as to correspond to wavelet dyadic decomposition values (i.e., powers of 2), and consecutive windows are chosen with an overlap of $T_{s}-1$ to avoid missing features that might be present at the window borders. The choice of window size can be used to strike a balance between a high level of transient compression and a resolution still sufficient to discriminate different original signal behaviors within the window.

For clarification on how the pre-processing actually works in practice, Figure 3 shows the evolution of a plant signal during a transient with a sampling rate of 30 seconds ( $N=56$ measurements are totally available).

Figure 3. Evolution of a generic plant signal during a transient.

The considered windows contain $T_{s}=16$ consecutive measurement times ( 8 minutes long in the application of this paper) and the overlap is 15 measurement time (7 minutes and 30 seconds). This means that a new wavelet transform is applied each time a new pattern is acquired from the process (30 seconds in the application of this paper).

Figure 4 shows the output of the pre-processing. Three data streams are generated from the original signal, at a rate equal to the original sampling rate ( 30 seconds). In the example of Figure 4 , an original data stream of $N=56$ patterns generates three data streams of 41 pattern each, since the first transformed pattern can be obtained only when 16 original patterns are available (after $7.5 \mathrm{~min}$, in this example ). Thus, the first value of the three coefficients refers to the time window from $t=0 \mathrm{~min}$ to $t=7.5 \mathrm{~min}$, the second to the time window from $t=0.5 \mathrm{~min}$ to $t=8$ min, and so on. 
Looking at the correspondence between the original signal and the pre-processing output, one can observe that the transformation produces a very compact, and yet very significant, description of the original signal. The mean residual feature reflects the general trend of the signal just as the average signal value within the analysis window would.

The value of the maximum coefficient reflects negative trends, step changes, and the negative components of spikes within the analysis window. In a dual way, the minimum coefficient reflects the positive trends, step changes, and positive components of spikes. For example, the observed big increase in the signal values between $t=0$ and $t=11$ min corresponds to a big (absolute) value of the minimum coefficient in the transformed coefficients from $t=7.5$ to $t=18.5 \mathrm{~min}$ and the small decrease in the signal value between $t=3.5 \mathrm{~min}$ and $t=4.5 \mathrm{~min}$ is matched by a small value of the maximum coefficient from $t=0$ to $t=12 \mathrm{~min}$. One can also appreciate how the transformation maintains the correct proportions of the registered changes.

Figure 4. Mean residual, maximum and minimum wavelet coefficients of the signal reported in Figure 3.

The approach here proposed for the signal reconstruction in transient conditions requires a preliminary phase in which the signal processing tool based on the Haar wavelet is applied to the training transients. Therefore, assuming a time window of $T_{s}$ patterns long and a window overlap of $T_{s}-1$ patterns, the application of the tool to the $N_{r}$ $J$-dimensional patterns of the $r$-th transient produces $N_{r}-T_{s}+1$ patterns, each one formed by $3 \cdot J$ features. Finally, the AAKR reconstruction model is built using as training set all the $3 \cdot J$-dimensional patterns obtained from the application of the preprocessing tool to all the available $R$ transients.

Then, in the test phase, one should (Figure 5):

1 apply the signal processing based on the Haar wavelet to the last $T_{s}=16$ available measurements $\vec{x}^{o b s}(t)$ of each of the $J$ signals. This generates a single $3 \cdot J$-dimensional test vector $\vec{y}(t)$;

2 reconstruct the obtained test vector $\vec{y}(t)$ by using the AAKR model, i.e., calculate $\overrightarrow{\hat{y}}(t)$. 
Figure 5. Sketch of Approach 2.

\section{Application}

A real case study concerning the condition monitoring of a gas turbine during start-up transients is considered. The available data contain the evolution of $J=115$ signals during $R=88$ start-up transients. The signal measurements have been collected every 30 seconds. Transients are of different time lengths varying from a minimum of $14.5 \mathrm{~min}$, during which $N_{r}=30$ measurement vectors have been collected, one every 30 seconds, to a maximum of 45 min corresponding to $N_{r}=91$ measurement vectors, with an average of $26.5 \mathrm{~min}$.

In [4] it has been shown that in industrial applications characterized by a high number of measured signals, grouping the signals and then building a specialized model for each group allows to remarkably increase the condition monitoring performance. With respect to the structure of the groups, different approaches have been proposed. In this work, we consider a grouping solution of the 115 measured signals provided by EDF engineers: signals have been divided into 6 groups containing signals measured in subsystems having the same function. A peculiarity of the proposed grouping is that there are few signals, such as the turbine shaft speed, that characterize the plant overall operation and are assigned to several groups at the same time. This grouping criterion has been shown in [21] to be effective for the reconstruction task.

The following analysis will be focused on Groups 1 and 4 which are the smallest and largest groups, respectively. In this respect, it has to be pointed out that according to [4], reconstruction models such as those based on AAKR tend to give more accurate reconstructions for groups formed by highly correlated signals and the best reconstructions are achieved for those signals characterized by the highest mean correlation with all other signals of the group. Therefore, since Group 1 is characterized by the presence of a subgroup of six highly correlated sig- 
nals and by the remaining two signals completely uncorrelated from all the others, these latter are eliminated from group 1.

In order to verify the performance of this approach, the following procedure has been applied. The 88 available transients have been randomly divided into a training and a test dataset. The training dataset contains $R=58$ transients (about $N_{t o t}=3100$ measurement vectors, since each transient is formed by several patterns), whereas the validation dataset contains the remaining 22 transients (about 1200 measurement vectors). The remaining 8 transients are used to find the optimal value of the parameter $h$ of the AAKR model (Appendix A), following a trial-anderror procedure [12]. Then, the metrics $M S E$ measuring the accuracy and $\overline{S_{a c}^{\text {auto }}}$ measuring the robustness have been computed on the validation set. The procedure is repeated 10 times according to a " 10 -fold" cross-validation error estimation scheme. The original dataset containing the 88 transients is randomly partitioned into 10 blocks, each one containing 8 or 9 transients; one of these blocks is used as validation data subset for the evaluation of the performance metrics of interest, and the remaining 9 blocks are combined together to constitute the training data and the data used to find the optimal model parameter values ( $h$ in the case of AAKR). The cross-validation process is then repeated 10 times (the 10 folds), each time using a different block as validation set.

\subsection{Approach 0: a single reconstruction model}

The AAKR reconstruction model is built using all the available data, independently from the time elapsed from the beginning of the start-up transient and the operational zone of the turbine. Table 2, column 1 reports the obtained results in terms of accuracy, robustness and computational time for the signals of groups 1 and 4 . The confidence intervals have been evaluated by using a 10-fold cross validation procedure.

The computational time tends to be quite long due to the large number of measurement vectors in the training set (about 3200), since each time a test vector is reconstructed, its distance to all the training measurement vectors has to be computed according to the AAKR reconstruction method.

Figure 6 shows the reconstruction of one of the signals, namely "401" (group 4), obtained for a transient in normal (upper-left) and abnormal (upper-right) conditions. Note that the residuals between the measurements and the reconstructions (Figure 6, middle-left) are close to zero in the normal condition case and, thus, the system is 
correctly recognized in normal conditions, whereas in the abnormal condition case, as expected, a fault is detected since the residuals between the measurements and the reconstructions (Figure 6, middle-right) remarkably deviate from zero. In this latter case, the results are also satisfactory in terms of robustness since the abnormal condition reconstructions are close to the measured signal values in normal conditions (Figure 6, bottom-right).

With respect to the normal condition reconstruction, notice, however, the oscillating behavior at time $t=5$ and 5.5 min. Similar oscillations have been observed also for the reconstructions of other 10 signals of Group 4.

Figure 6. Upper-left: real observed value of signal '401' (Group 4) during a transient in normal condition (continuous line) and obtained reconstruction (dotted line); upper-right: signal evolution in abnormal conditions (continuous line) and obtained reconstruction (dotted line); middleleft: residuals between the observed values and the reconstructions in case of normal conditions; middle-right: residuals between the observed values and the reconstructions in case of abnormal conditions; bottom-right: signal reconstructions in case of abnormal conditions (dotted line) and observed signal values in normal conditions (continuous line).

In order to investigate the causes of this oscillation in the reconstruction, Figure 7 shows the time evolution of some of the 58 start-up transients used to train the AAKR reconstruction model. When the vector containing the measurements taken at $t=4.5 \mathrm{~min}$ of the test transient in Figure 6 (left) is reconstructed, its multidimensional distance from all the training vectors is computed. The nearest training vector, which is the measurement vector with the associated highest weight in the AAKR reconstruction, belongs to the transient indicated by the thick black line. Notice that this transient is very similar to the test transient, and, thus, the reconstruction of the $t=4.5 \mathrm{~min}$ test vector is very close to the measured signal value. After $30 \mathrm{~s}$, when the vector containing the measurements taken at $t=5 \mathrm{~min}$ is considered for the reconstruction, the nearest training vector becomes a vector of a different training transient which is characterized by lower values of the signal, causing a reconstruction lower than the measured value. This change of the nearest training transient is not due to a variation of Figure 6 signal, whose values in the vectors at $t=4.5$ and $t=5 \mathrm{~min}$ are very similar, but to an abrupt variation of other signals such as signal ' 402 ' (Figures 8 and 9). Similarly, when the reconstruction of the vector at $t=5.5$ min is performed, the 
nearest training transient changes again becoming a transient characterized by higher values of Figure 6 signal. Also in this case, the cause is the remarkable variation of other signals such as that in Figure 8.

This oscillating effect in the reconstruction of signal ' 401 ' is related to the density of those training vectors that are closer to the test vector. As we can observe in Figure 7, the first part of the test transient is characterized by higher values of signal '401' (around 100) with respect to the majority of the transients characterized by values between 0 and 50. In this respect, notice that it has been verified that if we test a different transient whose signal '401' values between time $t=4.5$ and $5.5 \mathrm{~min}$ are around 15 , the oscillating behavior of the reconstruction would be reduced.

Figure 7. Blue continuous line: time evolution of signal ' 401 ' in some of the 58 training transients; large black lines: transients to which belong the vectors with the associated highest weight in the AAKR reconstruction of vectors 9, 10 and 11; dash-dotted black line: time evolution of the observed transient; dotted red line: reconstruction of the observed transient.

Figure 8. Time evolution of signal ' 402 ' in the 58 training transients.

Figure 9. Time evolution of signal ' 402 ' in the three transients containing the nearest vector to test vectors 9,10 and 11 (time $t=4.5,5$ and 5.5 min, respectively); thick line: evolution of the observed transient.

\subsection{Approach 1: a dedicated AAKR for each turbine operational zone}

This approach requires building a dedicated local model for each turbine operational zone. Operational zones have been defined according to signal ' 101 ' (belonging to both Groups 1 and 4) which is the pivot signal characterizing the start-up transients, according to EDF engineers. Table 1 reports the four zones considered in this work. 
Table 1. Definition of the turbine operational zones.

The training vectors have been split into four sets according to the value of signal ' 101 '. Each set has then be used for training a dedicated AAKR model. Notice that in the case in which one of the groups of signals does not contain the pivot signal, the dedicated AAKR models are still built considering a repartition of the historical measurement vectors based on the pivot signal. Then, during the test phase, the value of signal ' 101 ' in the test vector is compared with the limiting values of the four zones and the overall vector is sent to the corresponding AAKR reconstruction model.

Table 2 reports the obtained performances and their corresponding confidence intervals evaluated by using a 10-fold cross validation procedure. The results are compared to those obtained by a single AAKR model (Approach 0).

Table 2. Accuracy, robustness and total computational time obtained by using a single model (Approach 0 ) or by using a dedicated model for each operational zone (Approach 1). The confidence intervals are evaluated by using a 10-fold cross validation procedure.

Notice that Approach 1 is as accurate as Approach 0, but less robust. On the other hand, it is less time consuming, being the computational time about one third of that of Approach 0. This depends from the fact that the dataset used to train the AAKR model of Approach 0 is remarkably larger than the datasets used to train the AAKR models in Approach 1. Therefore, the number of distances between the test vector and the training vectors to be computed in Approach 0 is larger than that in Approach 1.

Table 3 reports the performance in the reconstruction of test vectors belonging to the different operational zones. The performances of the two approaches are very similar except for zone 4 where Approach 1 is remarkably less robust. This is mainly due to the fact that an abnormal condition which reduces signal ' 101 ' from a value higher than 98 to a value lower than 98 causes the change of the AAKR zone model used to reconstruct the vector (from zone 4 AAKR reconstruction model to zone 3 AAKR reconstruction model). Since zone 3 model is trained only 
with zone 3 vectors which are characterized by a signal ' 101 ' value lower than 98, zone 3 AAKR model is unable to reconstruct the signal '101' value in normal condition (a value larger than 98) and, thus, the approach is not robust to this type of disturbances. This effect is clearly shown in Figure 10: when the disturbance causes a lowering of the signal '101' value below 98 (Figure 10 top right, dotted line), the reconstruction with Approach 1 of the signal ' 101 ' value in normal condition becomes inaccurate (Figure 10, bottom right, dotted line), whereas the reconstruction provided by Approach 0 in the same condition is very accurate (Figure 10, bottom right, dasheddotted line).

Analogously, a disturbance applied to signal '101' which reduces the signal value below 98 causes inaccurate reconstructions of other signals (spill-over) due to the use of the zone 3 reconstruction model for vectors which, in normal conditions, would belong to zone 4.

Table 3. Accuracy and robustness in the four operational zones.

Figure 10. Left: real observed values of signal ' 101 ' during a transient in normal condition (continuous line) and obtained reconstruction (dotted line); upper right: simulated evolution of the signal in abnormal conditions; bottom right: signal reconstruction in case of abnormal conditions (dotted line) and its real value in normal conditions (continuous line).

Notice that different choices of the operational zones may lead to different performances of the approach. In particular, the fusion of zones 3 and 4 into a single zone may improve the robustness at the expenses of higher computational times. In generral,,$[$ itin $]$ s expected that larger is the number of operational zones, smaller is the computational time and the robustness. 
Approaches 0 and 1 perform the reconstruction of the present values of the signal measurements $\vec{x}^{o b s}(t)$ without taking into consideration the past evolution of the signals. This may lead to oscillating reconstructions when signals have abrupt changes such as those reported in Figure 6 (Approach 0) or the abnormal condition causes a variation of the pivot signal, as reported in Figure 10 (Approach 1). In order to overtake this limitation of Approaches 0 and 1, Approach 2 has been applied.

The window size, $T_{s}$ has been taken equal to 16 measurement times, which corresponds to 8 minutes, and the overlap is $\left(T_{s}-1\right)=15$ measurement times. This choice of the window size allows obtaining a satisfactory compression of the signals: following a trial-and-error procedure we have seen that a shorter widow $\left(T_{s}=8\right)$ do not consider enough information on the past evolution of the signals and, thus, produce reconstructions similar to that of approach 0 , whereas a longer window $\left(T_{s}=32\right)$ catches only the generic trends of the signals, neglecting signal oscillations occurring with smaller periods. With respect to the overlap, it has been chosen of 15 measurement times in order to avoid missing features that might be present at the window borders with shorter overlapping [10]. A new wavelet transform is applied each time a new measurement vector is acquired from the process ( 30 seconds in this application).

The application of Approach 2 to signal ' 101 ' in the start-up transient previously shown in Figure 10 (continuous line on the left refers to normal conditions, continuous line on the upper-right refers to abnormal conditions) produces the reconstructions shown in Figures 11 and 12 in the cases of normal and abnormal conditions, respectively. Notice the good agreement between the wavelet coefficients of signal '101' in normal conditions and their wavelet reconstructions in both cases. This indicates the accuracy and robustness of the method, i.e. its capability of properly estimates the expected values of the wavelet coefficients.

Since the anti-transformation from the wavelet domain to the time domain requires the availability of all the Haar coefficients of the reconstruction, the proposed approach does not allow to obtain the signal reconstruction in the time domain. This implies that Approach 2 cannot be evaluated from the point of view of the overall accuracy and robustness by applying the metrics of Appendixes B and C to the signal reconstruction in the time domain. On the contrary, the comparison can be made by computing the performance metrics in the $3 \cdot J$ dimensional space of 
the wavelet transform. In other words, the MSE and $S_{\text {auto }}$ are evaluated according to Eqs. B2 and C2, respectively, where instead of $x^{\text {test-nc }}(k, j)$ we consider the wavelet transform of the $j$-th signal performed by considering signal $j$ values at the measurement times $k-T_{s}+1, k-T_{s}+2, \ldots, k$ of the test transient in normal condition, instead of $x^{\text {test }-a c}(k, j)$ the wavelet transform of the same signal $j$ values in abnormal condition, and instead of $\hat{x}_{n c}^{\text {test }}(k, j)$ the reconstructions of the obtained wavelet coefficients.

This comparison requires applying the signal processing tool based on the Haar wavelet also to the reconstructions obtained by Approaches 0 and 1. Table 4 compares the performances obtained by the three different approaches. Since the metrics in Table 4 are computed on the wavelet transform space, their absolute values for approaches 1 and 4 are different.

Figure 11. Wavelet coefficients of signal '101'during the normal condition transient considered in Figure 10-left, (continuous line) and obtained reconstruction (dotted line); top: mean residual; center: maximum coefficient; bottom: minimum coefficient.

Figure 12. Dotted line: AAKR reconstruction of the wavelet coefficients in the simulated abnormal condition (Figure 10 upper-left, continuous line); Continuous line: wavelet coefficient value in normal condition. Top: mean residual; center: maximum coefficient; bottom: minimum coefficient. In the last two Figures, the ordinate axis scale has been enlarged in order to better show the results.

Table 4. Accuracy and robustness metrics and computational time obtained by using a single model (Approach 0 ), by using a dedicated model for each operational zone (Approach 1), or the wavelet transform data pre-processing (Approach 2).

Notice that Approach 2 is less accurate but more robust than Approaches 0 and 1. From the point of view of the computational efforts, Approach 2 is more burdensome than Approach 1 but less than Approach 0. Notice that the metrics values reported in Table 4 are different from those provided in Table 2 since they refer to the Haar transforms and not to the signal values. A possible reason of the lower accuracy of Approach 2 is the larger number of 
features which are given in input to the reconstruction model. As it has been shown in [4,21], larger is the number of features in a group, lower tend to be its accuracy. A possibility to improve Approach 2 accuracy is to search for optimal subgroups in the 147 features of group 4 and then developing a dedicated AAKR model for each subgroup $[4,22]$. Notice also that the subdivision of the signals in more subgroups would lead to a further reduction of the computational times.

Finally, we have tested Approach 2 with the test transient of Figure 8. Figure 13 compares the reconstruction of the wavelet coefficients obtained using Approach 2 with the wavelet transform of the signal reconstruction provided by Approach 0. Notice that:

- Approach 2 is less accurate with respect to the mean residual given that it tends to underestimate the correct signal value;

- the oscillation in the signal reconstruction provided by Approach 0 causes anomalous values of the minimum and maximum wavelet coefficients obtained from Approach 0. Approach 2 reconstructions do not show these anomalous values of the minimum and maximum wavelet coefficients and are very similar to the corresponding wavelet obtained from the measured signal.

These results confirms the conclusions given in Table 4 according to which Approach 2 is less accurate but more robust than Approach 0.

Figure 13. Wavelet coefficients of the real observed values (continuous line), the Approach 0 reconstruction ('- $\mathrm{x}-{ }^{\text {' }}$ line) and the Approach 2 reconstruction (dotted line); top Figure: mean residual; middle Figure: maximum coefficient; bottom Figure: minimum coefficient.

\section{Conclusions}

In this paper we have addressed the issue of the detection of abnormal component conditions during plant transients such as start-up and shut-down. To this aim, we have developed two novel approaches for signal reconstruction. A case study regarding the condition monito Time [min] rbines start-up transients has been considered. The performance of a traditional AAKR model developed considering as training set all the available measure- 
ment vectors (Approach 0), independently from the time elapsed from the beginning of the transient and the turbine speed operational zone, seems to give satisfactory performance in terms of the overall accuracy and robustness. The two main drawbacks of the approach are: a) the high computational time required for its online application ,which is due to the computation of the distances between the current measurements and all the historical measurement vectors in the training set; b) the low accuracy and tendency to oscillate of the reconstructions in correspondence of test vectors which belong to zones of the training space characterized by a low density of measurement vectors. These oscillations have been noticed especially in correspondence of abrupt changes in some signals values.

With respect to the first limitation, a possible solution is to develop different AAKR reconstruction models, each one dedicated to a different turbine operational zone (Approach 1). However, although the computational effort has been remarkably reduced by using different models, the robustness and resistance of this approach in correspondence of vectors at the borders between the different operational zones have not been always satisfactory.

In order to reduce the inaccuracy of the signal reconstruction in correspondence of abrupt modifications of some measured signals, an approach which takes into account not only the present value but also the past evolution of the signals has been considered (Approach 2). To this purpose, the use of a signal processing tool based on the Haar wavelet transform has been proposed. The approach has been shown to be able to provide robust reconstruction in case of abrupt signal changes, although it tends to be slightly less accurate in the reconstruction of smooth transients. This is due to the larger number of features which are given in input to the reconstruction model by Approach 2, since for ach feature, it consider 3 wavelet coefficients. A possibility to improve Approach 2 accuracy is to search for optimal subgroups by using optimization algorithm, such as Genetic Algorithms and then developing a dedicated AAKR model for each subgroup.

Finally, notice that once the signal reconstruction task is performed, statistical methods of analysis of the deviations between the observed and reconstructed values, such as the Sequential Probability Ratio Test (SPRT) can be applied for fault detection and alarm triggering. The application of these methods during plant operational transients will be object of future research work. 


\section{Acknowledgements}

This research has been carried out under contract EDF/PDM C935C50010 "Processing condition monitoring data for diagnosis and prognosis of components of electricity production plants“ funded by EdF R\&D. The authors wish to thank the anonymous reviewers for their constructive comments and suggestions which have allowed improving the the paper.

\section{Appendix A: the Auto-Associative Kernel Regression (AAKR) method}

The basic idea of the AAKR method is to perform the reconstruction of the current signal measurements, $\vec{x}^{o b s}(t)=\left[x^{o b s}(1), x^{o b s}(2), \ldots, x^{o b s}(J)\right]$, on the basis of historical signal observations. Notice that, for the sake of simplicity, the current time $t$ is not reported in the notation $x^{o b s}(j)$ of the value of signal $j$ measured at time $t$. Let us indicate with $X^{o b s-n c}$ a matrix whose generic $k$-th row, hereafter indicated by $\vec{x}^{o b s-n c}(k), k=1, \ldots, N$, contains the $J$ signal values measured at the observation time $t_{k}$.

The reconstruction 资 $(t)=\left[x_{n c}(1), x_{n c}(), \ldots, x_{n c}(J)\right]$ at the current time $t$ of the $J$ measured signal is performed as a weighted sum of the historical signal observations in $\overline{\bar{X}}^{o b s-n c}$. In particular, the reconstruction $\hat{x}_{n c}(j)$ of the value of signal $j$ at the present time $t$ is given by:

$$
\left.\chi_{n c} \quad j\right)=\frac{\sum_{k=1}^{N} w(k) \cdot x^{o b s-n c}(k, j)}{\sum_{k=1}^{N} w(k)}
$$

The weights $w(k)$ are similarity measures obtained by computing the normalizd Euclidean distance between the current sensor measurements $\vec{x}^{o b s}(t)$ and $\vec{x}^{o b s-n c}(k)$ : 


$$
d^{2}(k)=\sum_{j=1}^{j} \frac{\left(x^{o b s}(j)-x^{o b s-n c}(k, j)\right)^{2}}{\sigma_{j}^{2}}(A 2)
$$

The signal variance at the denominator $\sigma_{j}^{2}=\frac{\sum_{k=1}^{N}\left(x^{o b s-n c}(k, j)-\mu(j)\right)^{2}}{N-1}$ with $\mu(j)=\frac{\sum_{k=1}^{N} x^{o b s-n c}(k, j)}{N}$ the signal mean value has been introduced in order to provide a common scale across the different signals measuring different quantities. Finally, the computed distances are inserted in the Gaussian kernel:

$$
w(k)=\frac{1}{\sqrt{2 \pi} h} e^{-\frac{d^{2}(k)}{2 h^{2}}}
$$

where $h$ defines the Gaussian kernel bandwidth which controls how fast the function is damped, i.e., the smaller $h$ the smaller the weights of those observations of $X^{\text {obs-nc }}$ characterized by large distances $d^{2}(k)$.

\section{Appendix B: Accuracy metric}

In order to evaluate the performance of the reconstruction method, we consider a matrix $\overline{\bar{X}}^{\text {test-nc }}$ containing historical signal measurements taken during normal plant condition different from those in $\overline{\bar{X}}^{\text {obs-nc}}$ used by the AAKR method to perform the reconstruction. In practice, all the available historical signal measurements are divided into a set $\overline{\bar{X}}^{\text {obs-nc }}$ made by $N$ measurement vectors used by for the AAKR reconstruction and a set $\overline{\bar{X}}^{\text {test-nc }}$ made by $N^{\text {test }}$ measurement vectors used for evaluating the method performance. Indicating by $x^{\text {test-nc }}(k, j)$ the true value of the $j$ th signal, $j=1, \ldots, J$, at the $k$-th observation time, $k=1, \ldots, N^{\text {test }}$ and by $\hat{x}_{n c}^{\text {test }}(k, j)$ its AAKR reconstruction, the mean square error with respect to signal $j$ is:

$$
\operatorname{MSE}(j)=\frac{\left.\sum_{k=1}^{N^{\text {test }}}\left(x_{n c}^{\text {test }} k, j\right)-x^{\text {test }-n c}(k, j)\right)^{2}}{N^{\text {test }}}
$$


A global accuracy measure that takes into account all the monitored signals and test vectors is defined by:

$$
M S E=\frac{\sum_{j=1}^{J} \sum_{k=1}^{N^{\text {test }}} \frac{\left.\left(\mathcal{X}_{n c}^{\text {est }} k, j\right)-x^{\text {test } n c}(k, j)\right)^{2}}{N^{t e s t}}}{J}=\frac{\sum_{j=1}^{J} M S E(j)}{J}
$$

Notice that, although the metric is named accuracy, it is actually a measure of error and, thus, a low value is desired.

\section{Appendix C: Robustness metrics}

Let $X^{\text {test-ac }}$ be a matrix of test vectors obtained from $\bar{X}^{\overline{t e s t}-n c}$ by adding to it a disturb in order to artificially reproduce an abnormal condition. Indicating by $x^{\text {test-ac }}(k, j)$ the value of the $j$-th signal at the $k$-th observation time in the simulated abnormal conditions, $k=1, \ldots, N^{\text {test }}$, and $\hat{x}^{\text {test }}(k, j)$ its AAKR reconstruction, which is expected to be the signal value in normal condition $x^{\text {test-nc }}(k, j)$, quantitative indicators of robustness can then be introduced as follows.

The auto-sensitivity of the model to a disturbance applied on signal $j$ is defined as:

$$
S_{a c}^{\text {auto }}(j)=\frac{1}{N^{\text {test }}} \sum_{k=1}^{N^{\text {test }}}\left|\frac{\left.\left.\mathscr{X}^{\text {test }} k, j\right)-\mathcal{X}_{n c}^{\text {est }} k, j\right)}{x^{\text {test }-a c}(k, j)-x^{\text {test }-n c}(k, j)}\right|
$$

This metric measures the ability of the model to provide the same reconstructions in the two cases of disturbed or undisturbed signal $j$. In this respect, notice that a model characterized by a very low accuracy (large MSE) and very high robustness (small $S_{a c}^{\text {auto }}(j)$ ) is not satisfactory for condition monitoring since it still provides signal reconstructions very different from signal values in normal plant operation.

A global robustness measure $\overline{S_{a c}^{\text {auto }}}$ is then obtained by applying a disturbance to all the signals, computing the robustness $S_{a c}^{\text {auto }}(j)$ and taking the mean value: 


$$
\overline{S_{a c}^{\text {auto }}}=\frac{\sum_{j=1}^{J} S_{a c}^{\text {cuto }}(j)}{J}
$$

Since real data in abnormal plant conditions are not available, simulation is used to artificially inject abnormality by adding a random noise to the signal measurements taken in normal plant conditions.

\section{References}

[1] J. Reifman, 'Survey of artificial intelligence methods for detection and identification of component faults in nuclear power plants', Nucl. Technol., 119, pp 76-97, 1997.

[2] P. Vaidya, M. Rausand, "Remaining useful life, technical health, and life extension", Proceedings of the Institution of Mechanical Engineers, Part O, Journal of Risk and Reliability, 225 (2), pp. 219-231, 2011

[3] P. Baraldi, M. Compare, A. Despujols, E. Zio, "Modeling of the effect of maintenance actions on the degradation of an electric power plant component". Proceedings of the Institution of Mechanical Engineers, Part O, Journal of Risk and Reliability, 225 (2), pp. 169-184, 2011

[4] P. Baraldi, R. Canesi, E. Zio, R. Seraoui and R. Chevalier, 'Signal Grouping for Condition Monitoring of Nuclear Power Plant Components', Seventh American Nuclear Society International Topical Meeting on Nuclear Plant Instrumentation, Control and Human-Machine Interface Technologies NPIC\&HMIT 2010, Las Vegas, Nevada, November 7-11, 2010.

[5] J. W. Hines and D. R. Garvey, 'Development and Application of Fault Detectability Performance Metrics for Instrument Calibration Verification and Anomaly Detection', Journal of Pattern Recognition Research, 1, pp 2-15, 2006.

[6] Strang, G. \& Nguyen, T., Wavelets and Filter Banks, Wellesley-Cambridge Press, 1996.

[7] R. T. Ogden, “Essential Wavelets for Statistical Applications and Data Analysis”, Birkhausen, Boston, 1997.

[8] M. Vetterli, C. Herley, “Wavelets and Filter Banks: Theory and Design", IEEE Transactions on Signal Processing, 40(9), 2207-2231, 1992.

[9] D. Roverso, "Fault diagnosis with the aladdin transient classifier." In: Proceedings of System Diagnosis and Prognosis: Security and Condition Monitoring Issues III, AeroSense2003, Aerospace and Defense Sensing and Control Technologies Symposium. Orlando, FL, 21-25 April 2003.

[10]D. Roverso, "Soft computing tools for transient classification", Information Sciences, 127, pp 137-156, 2000.

[11]P. Baraldi, N. Pedroni, E. Zio, “Application of a Niched Pareto Genetic Algorithm for Selecting Features for Nuclear Transients Classification”, International Journal of Computational Intelligence Systems, Vol. 24 (2), pp. 118-151, 2009.

[12]P. Baraldi, R. Canesi, E. Zio, R. Seraoui, R. Chevalier, "Generic algorithm-based wrapper approach for grouping condition monitoring signal of nuclear power plant components". Integrated Computer-Aided Engineering, Vol. 18 (3), pp. 221-234, 2011. 
[13] K.C. Gross and K.E. Kumenik, 'Sequential probability ratio test for nuclear power plant component surveillance’, Nuclear Technology 93, pp. 131-137, 1991.

[14]K. C. Gross, W. Lu, "Early Detection of Signal and Process Anomalies in Enterprise Computing Systems", International Conference on Machine Learning and Applications - ICMLA - 204-210, 2002.

[15]E. Zio, P. Baraldi and N. Pedroni, 'Selecting Features for Nuclear Transients Classification by means of Genetic Algorithms', IEEE Transactions on Nuclear Science, 53, pp. 1479-1493, 2006

[16]B. Efron, 'Estimating the error rate of a prediction rule: Improvement on cross-validation', Journal of the American Statistical Association, 78, pp. 316-331, 1983

[17]B. Efron and R.J. Tibshirani, 'Improvements on cross-validation: The .632+ bootstrap method', Journal of the American Statistical Association, 92, pp. 548-560, 1995.

[18] R. Kohavi, 'A Study of Cross-Validation and Bootstrap for Accuracy Estimation and Model Selection', San Mateo, CA: Morgan Kaufman. pp. 1137-1143, 1995.

[19] R. Polikar, 'Bootstrap-Inspired Techniques in Computational Intelligence’, IEEE signal processing magazine, 59, pp. 59-72, 2007.

[20]D. Roverso, "Multivariate Temporal Classification by Windowed Wavelet Decomposition and Recurrent Neural Networks", International Topical Meeting on Nuclear Power Plant Instrumentation, Controls and Human-Machine Interface Technologies (NPIC\&HMIT2000), Washington, DC, November, 2000.

[21]P. Baraldi, E. Zio, F. Di Maio, L. Pappaglione, R. Chevalier, R. Seraoui, “Differential Evolution for Optimal Grouping of Condition Monitoring Signals of Nuclear Components", Proceedings of ESREL 2011 Conference, pp. 410-418, Troyes, France, 18-22 September, 2011.

[22]P. Baraldi, E. Zio, G. Gola, D. Roverso, M. Hoffmann, “Genetic Algorithms for Signal Grouping in Sensor Validation: a Comparison of the Filter and Wrapper Approaches", Proceedings of the Institution of Mechanical Engineers, Part O: Journal of Risk and Reliability, Vol. 222 (2), pp. 189-206, 2008.

[23]E. Zio, P. Baraldi and G. Gola, "Ensemble feature selection for diagnosing multiple faults in rotating machinery", Proceedings of the Institution of Mechanical Engineers, Part O: Journal of Risk and Reliability, Vol. 221 (O1), pp. 29-41, 2007. 